\title{
A statistical study on the shape and position of the magnetotail neutral sheet
}

\author{
Sudong Xiao ${ }^{1}$, Tielong Zhang ${ }^{1,2}$, Yasong Ge ${ }^{3}$, Guoqiang Wang ${ }^{1}$, Wolfgang Baumjohann ${ }^{2}$, and Rumi Nakamura ${ }^{2}$ \\ ${ }^{1}$ CAS Key Laboratory of Geospace Environment, University of Science and Technology of China, Hefei, China \\ ${ }^{2}$ Space Research Institute, Austrian Academy of Sciences, Graz, Austria \\ ${ }^{3}$ Institute of Geology and Geophysics, Chinese Academy of Sciences, Beijing, China \\ Correspondence to: Tielong Zhang (tielong.zhang@oeaw.ac.at)
}

Received: 2 July 2015 - Revised: 17 January 2016 - Accepted: 10 February 2016 - Published: 26 February 2016

\begin{abstract}
We study the average shape and position of the magnetotail neutral sheet based on magnetic field data obtained by Cluster, Geotail, TC-1, and THEMIS from the years 1995 to 2013. All data in the aberrated GSM (geocentric solar magnetospheric) coordinate system are normalized to the same solar wind pressure $2 \mathrm{nPa}$ and downtail distance $X \sim-20 R_{\mathrm{E}}$. Our results show characteristics of the neutral sheet, as follows. (1) The neutral sheet assumes a greater degree of curve in the $Y Z$ cross section when the dipole tilt increases, the Earth dipole tilt angle affects the neutral sheet configuration not only in the $Y Z$ cross section but also in the $X Y$ cross section, and the neutral sheet assumes a more significant degree of tilt in the $X Y$ cross section when the dipole tilt increases. (2) Counterclockwise twisting of the neutral sheet with $3.10^{\circ}$ is observed, looking along the downtail direction, for the positive interplanetary magnetic field (IMF) $B_{Y}$ with a value of 3 to $8 \mathrm{nT}$, and clockwise twisting of the neutral sheet with $3.37^{\circ}$ for the negative IMF $B_{Y}$ with a value of -8 to $-3 \mathrm{nT}$, and a northward IMF can result in a greater twisting of the near-tail neutral sheet than southward. The above results can be a reference to the neutral sheet model. Our large database also shows that the displaced ellipse model is effective to study the average shape of the neutral sheet with proper parameters when the dipole tilt angle is larger (less) than $10^{\circ}\left(-10^{\circ}\right)$.
\end{abstract}

Keywords. Magnetospheric physics (magnetotail)

\section{Introduction}

The neutral sheet of the magnetotail is located in the middle of the plasma sheet, lying between two lobes (Ness, 1965; Baumjohann and Treumann, 2012). It is characterized by a weak magnetic field, strong cross tail current, and a reversal of the magnetic field direction across it. The neutral sheet is important for the formation of the magnetotail, and the dynamics of the Earth's magnetosphere are greatly influenced by physical processes that occur near the neutral sheet. The exact position of the neutral sheet is variable with time. The shape of the neutral sheet is known to be warped because of the tilting of the Earth's magnetic dipole. Figure 1 illustrates the neutral sheet elliptical shape as controlled by the dipole tilt angle, $\chi$. The dipole tilt is defined as the angle between the Earth's north dipole axis and the $Z$ axis in the GSM (geocentric solar magnetospheric) coordinate system and is positive when the north magnetic pole is tilted toward the Sun.

Therefore, it is essential to have a reliable estimate of the average position of the neutral sheet. Several shape models of the neutral sheet are proposed in early studies. For example, a step model is proposed by Murayama (1966), and a standard ellipse model is proposed by Russell and Brody (1967). While the step model stands against theory and observation, the standard ellipse model gives unequal cross-sectional areas for north and south lobes. That would yield different average magnetic field magnitudes in the two lobes, inconsistent with the observations.

To obtain an observational model of the neutral sheet, three major studies have been done in the last several decades, i.e., Fairfield (1980), Hammond et al. (1994), and Tsyganenko and Fairfield (2004). Fairfield (1980) proposed a displaced 


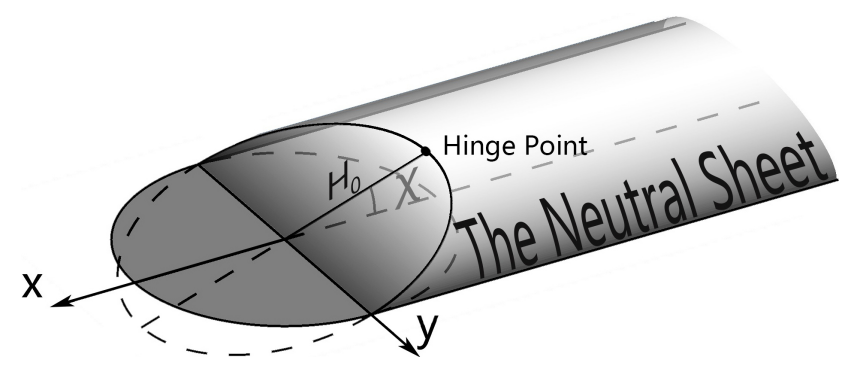

Figure 1. A 3-D diagram of the magnetotail neutral sheet. The sketch illustrates the geomagnetic neutral sheet as the tailward projection of an ellipse which is tilted at angle $\chi$, which is the dipole tilt angle, to the solar magnetospheric plane. The solid line is above the plane and the dotted line is under the plane. The angle between the $X Y$ plane and the magnetic equatorial plane also equals the dipole tilt angle $\chi$. The hinging point is indicated in this figure, and the hinging distance $H_{0}$ is the distance from the Earth's center to the hinging point, as shown in this figure.

ellipse neutral sheet model as shown in Fig. 2, given by

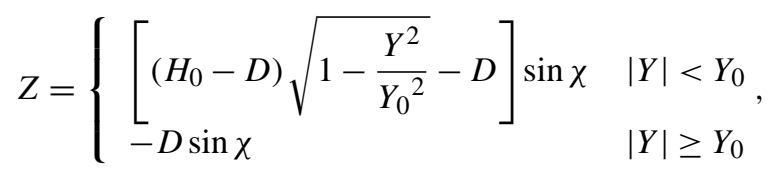

where $H_{0}$ is the magnetotail hinging distance from the Earth to the hinge point shown in Fig. $1, D$ is a factor of the displacement of ellipse, and $Y_{0}$ is the semi-major axis of the model ellipse, as Fig. 2 shows. This model accounts for asymmetrical areas of north and south lobes via a displacement along $Z$. For eliminating the aberration effect caused by the Earth's orbital motion, also called the windsock effect, all data were rotated to the aberrated GSM (AGSM) coordinate system. The AGSM coordinate system has its $X$ axis antiparallel to the average direction of the solar wind, aberrated from the Sun-Earth line. As with the GSM coordinate system, the $X Y$ plane contains the Earth magnetic dipole axis with a $Z$ axis chosen to be in the same sense as the northern magnetic pole, and the $Y$ axis completes the right-handed Cartesian coordinate system.

Similar to Fairfield (1980), Hammond et al. (1994) also chose the displaced ellipse neutral sheet model and the AGSM coordinate system, but with a more exact aberration angle of $4^{\circ}$. In addition, all data in the magnetosphere were normalized to the same solar wind dynamic pressure $(3.8 \mathrm{nPa})$ using OMNI solar wind data with the equation of $R$, the magnetotail radius, given as

$R \propto P_{\mathrm{obs}}^{-\frac{1}{6}}$

and a cross section $\left(X=-25 R_{\mathrm{E}}\right)$ given by the flaring magnetopause model equation of Howe Jr. and Binsack (1972) as

$$
R \propto \arctan \left(\sqrt{\frac{10-X_{\mathrm{AGSM}}}{15.9}}\right) .
$$

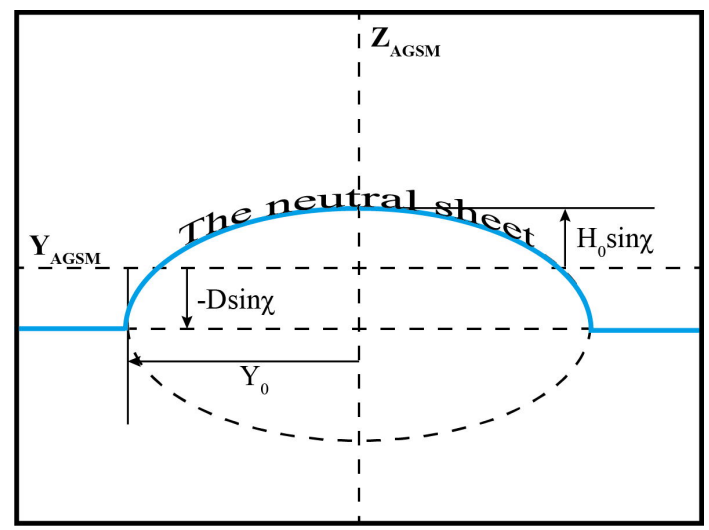

Figure 2. The displaced ellipse neutral sheet model shown on the cross section of the magnetotail. The blue line indicates the neutral sheet. The parameters of the model are marked in this figure and "-Dsin $\chi$ " is the displaced degree.

Different from Fairfield (1980), Hammond et al. (1994) counted data to bins on a cross section, and then obtained parameters via fitting the points where the $X$ component of the magnetic field changes sign. Tsyganenko and Fairfield (2004) developed an analytical approximation for the shape of the nightside tail current sheet with a semi-empirical model as a function of the Earth's dipole tilt angle, solar wind ram pressure, and the interplanetary magnetic field (IMF). The model of Tsyganenko and Fairfield (2004) was developed for covering a downtail distance from 0 to $50 R_{\mathrm{E}}$, containing the region of the dipolar magnetic field.

The shape of the neutral sheet can be greatly affected by the solar wind parameters, the IMF, and the dipole tilt angle (Russell, 1972; Sibeck et al., 1985; Tsyganenko and Fairfield, 2004). Russell $(1972,1973)$ predicted that the IMF $B_{Y}$ can twist the magnetotail neutral sheet. Sibeck et al. (1985) found that the neutral sheet can be twisted left- or righthanded by the positive or negative IMF $B_{Y}$, respectively. Further observations found that a northward IMF can result in a greater twisting (e.g., Owen et al. 1995). Later, Tsyganenko and Fairfield (2004) found that an increase in solar wind dynamic pressure results in a decrease in the hinging distance $H_{0}$. Recently, based on the model of Tsyganenko and Fairfield (2004), Tsyganenko et al. (2015) developed a new quantitative model of the shape of the magnetospheric equatorial current sheet as a function of the dipole tilt angle, solar wind dynamic pressure, and IMF, and the model covers all local time, including the dayside sector.

A mass of data can now be obtained by spacecraft, such as Cluster, TC- 1 and THEMIS, and a new comprehensive and intuitive observation of the magnetotail neutral sheet is expected. In this study, we investigate the average shape of the neutral sheet affected by the dipolar tilt angle, the neutral sheet configuration in the $X Y$ cross section, and the IMF twisting effect on the neutral sheet. 

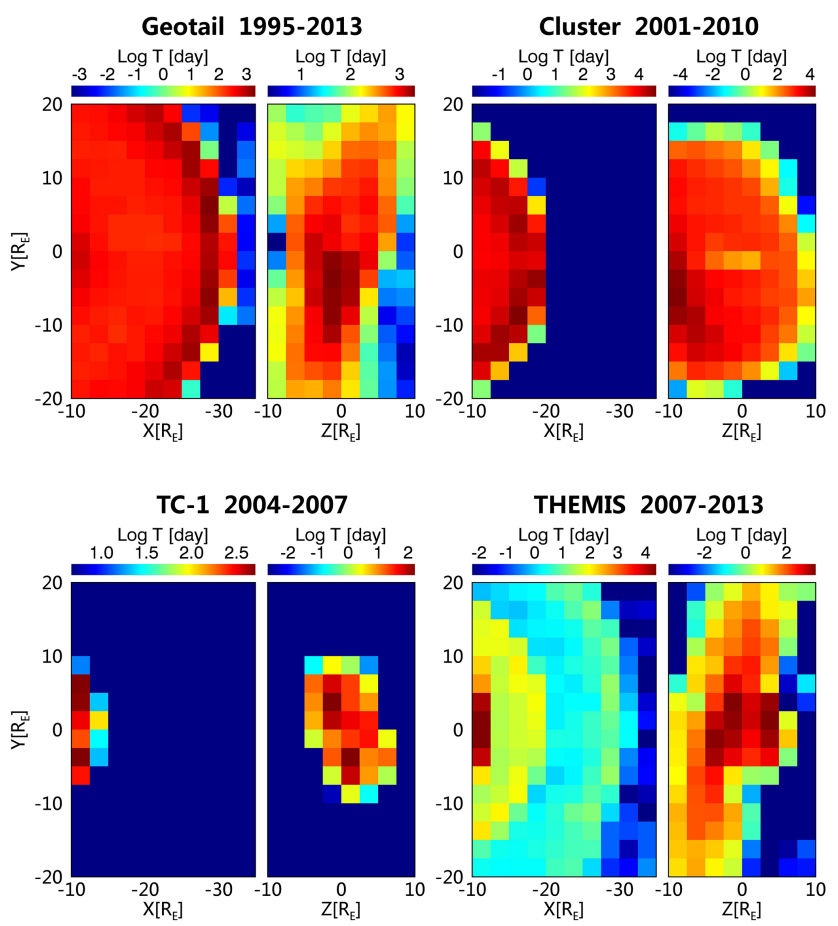

Figure 3. Orbital coverages of Geotail during the 19-year (19952013) tail period, Cluster 1-4 during the 10-year (2001-2010) tail period, TC-1 during the 4-year (2004-2007) tail period, and THEMIS P1-P5 during the 7-year (2007-2013) tail period. The bin size is $2.5 R_{\mathrm{E}} \times 2.5 R_{\mathrm{E}}$. In each group, the left image indicates the orbital coverage in the $X Y$ plane and the right panel indicates the orbital coverage in the $Z Y$ plane. The residence times of the satellites are given in days.

\section{Data and methods}

More data and satellites have become available since the work of Tsyganenko and Fairfield (2004). In total, 10 years (2001-2010) of Cluster 1-4 (Balogh et al., 2001), 19 years (1995-2013) of Geotail (Kokubun et al., 1994), 4 years (2004-2007) of TC-1 (Carr et al., 2005), and 7 years (20072009 for P1/P2; 2007-2013 for P3-P5) of THEMIS (Auster et al., 2008) magnetometer data are used in our study, with an original resolution of $4,64,60$, and $3 \mathrm{~s}$, respectively. The data from Cluster and THEMIS are averaged over $1 \mathrm{~min}$ intervals. In addition, the concurrent solar wind and IMF data from OMNI (5 min resolution) are combined to normalize the magnetotail neutral sheet model. To obtain symmetric images, the data are converted into the AGSM coordinate system with an average aberration angle of $4^{\circ}$.

This work focuses on the near-Earth and middle tail. The hinging distance can serve as an indication of the range of the dipolar magnetic field of the Earth. Based on prior studies, the hinging distance is less than $10 R_{\mathrm{E}}$, so we consider the area with a downtail distance beyond $10 R_{\mathrm{E}}$. Furthermore, in consideration of the satellite orbits, the observation

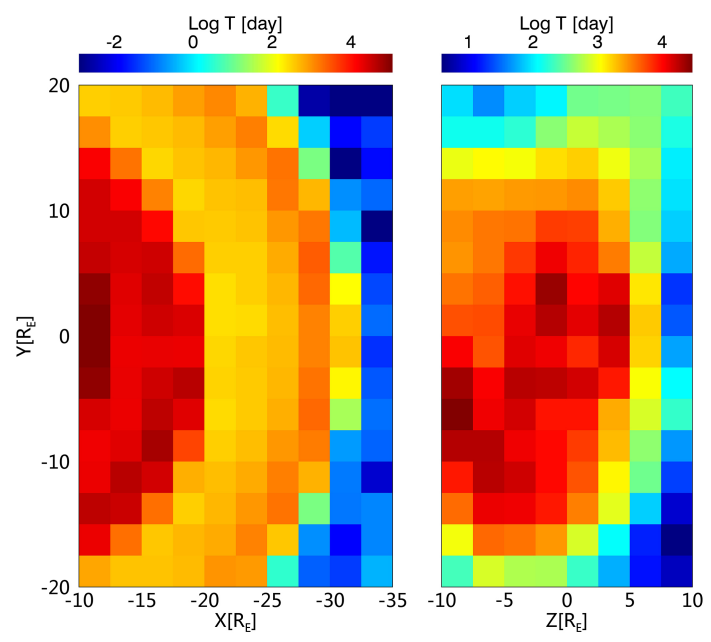

Figure 4. Orbital coverage of all satellites in the $X Y$ and $Z Y$ plane in the AGSM coordinate system. The bin size is $2.5 R_{\mathrm{E}} \times 2.5 R_{\mathrm{E}}$. The left panel indicates the orbital coverage in the $X Y$ plane and the right panel indicates the orbital coverage in the $Z Y$ plane. As this figure shows, a better coverage in space and time can be obtained by merging the data of different satellites.

region is set as $-35 R_{\mathrm{E}}<X<-10 R_{\mathrm{E}},-20 R_{\mathrm{E}}<Y<20 R_{\mathrm{E}}$, and $-10 R_{\mathrm{E}}<Z<10 R_{\mathrm{E}}$ (in AGSM, which is used throughout the paper unless mentioned otherwise). Figure 3 shows orbit coverage of the magnetic field data obtained from Geotail, Cluster, TC-1, and THEMIS satellites projected onto the $X Y$ and $Y Z$ plane with bin size $2.5 \times 2.5 R_{\mathrm{E}}$. One can find that THEMIS and Geotail have a large number of data and extensive spatial coverage. Cluster provides a large number of data, but the coverage is concentrated in the area with a near-to-middle tail distance. The orbit of TC-1 is lower than Cluster, providing more data nearer to the Earth.

In order to combine all data from different satellites to study the average shape and position of the neutral sheet, the data should be merged into one unified data set. Similar to Fig. 3, the unified data set is plotted in the $X Y$ (left panel) and $Y Z$ (right panel) plane. As shown in Fig. 4, combining the data from different satellites provides better coverage, in space and time.

The data are normalized to the same conditions to eliminate the effect of the solar wind dynamic pressure and the flaring of the magnetotail with the same method used by Hammond et al. (1994). The solar wind dynamic pressure corresponding to the magnetic data used in this study is shown in Fig. 5a. The mean value of the solar wind dynamic pressure is $\sim 2 \mathrm{nPa}$. Thereby, all data are normalized to a reference solar wind dynamic pressure of $2 \mathrm{nPa}$. As a reference, Fig. 5b shows the statistics of the solar wind dynamic pressure data obtained from OMNI for years 1995 to 2013, which also have a mean value of $\sim 2 \mathrm{nPa}$. Because of the flaring of the magnetotail, the cross section varies with downtail distance, even at fixed solar wind dynamic pressure. In order 

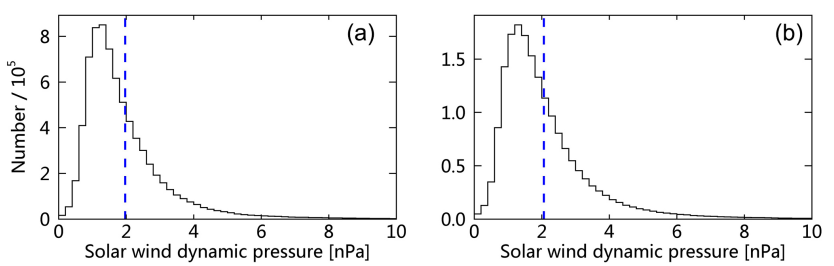

Figure 5. Histograms of the solar wind dynamic pressure. The panels show the results of the concurrent solar wind dynamic pressure when the magnetosphere data are available and analyzed in this study (a) and the OMNI data spanning the years 1995-2013 (b). The blue dashed lines show the mean values of the solar wind dynamic pressure, at about $2 \mathrm{nPa}$.

to eliminate this effect, all data are normalized to the same downtail distance with the magnetopause model of Howe Jr. and Binsack (1972). Considering the data distribution from $X \sim-10 R_{\mathrm{E}}$ to $X \sim-35 R_{\mathrm{E}}$, a reference value of a downtail distance of $20 R_{\mathrm{E}}$ is chosen in this study.

The next step is to obtain images of the cross section of the magnetotail containing the average shape and position of the neutral sheet. In order to investigate the effect of the dipole tilt on the neutral sheet, the data are divided into 14 sets with a dipole tilt angle interval of $5^{\circ}$. Magnetic field data in each set are binned into $0.5 \times 0.5 R_{\mathrm{E}}$ bins in a plane of $|Y|<20 R_{\mathrm{E}}$ and $|Z|<10 R_{\mathrm{E}}$. Bins are ignored if they contain fewer than 10 data points. We count the number of data with a positive $B_{X}$ in each bin and calculate the percentage. A percentage of more than $50 \%$ indicates that $B_{X}$ in the corresponding bin is positive, and a percentage of less than $50 \%$ indicates a negative $B_{X}$.

\section{Global shape of the magnetotail neutral sheet}

Figure 6 shows the shape of the neutral sheet under different dipole tilt angle. Red indicates that $B_{X}$ is positive and blue indicates negative $B_{X}$. The position between the two colors is considered the neutral sheet. As Fig. 6 shows, the neutral sheet is curved more with increasing dipole tilt. The neutral sheet curves toward north for positive dipole tilts and toward south for negative dipole tilts. The correlation between the dipole tilt and the curvature of the neutral sheet can be expressed as a sine function. The points where the sign of $B_{X}$ changes are recorded in Fig. 7, and the points where data are lacking are ignored. Our study focus on the average shape and position of the neutral sheet; therefore an empirical model of the displaced ellipse model is chosen to fit the shape of the neutral sheet. The average position and shape of the neutral sheet can be parameterized by fitting these points using the displaced ellipse model. The fitted curves are also plotted in Fig. 7. We calculate the correlation coefficient $\left(R^{2}\right)$ for each best-fitted curve, labeled on each panel.
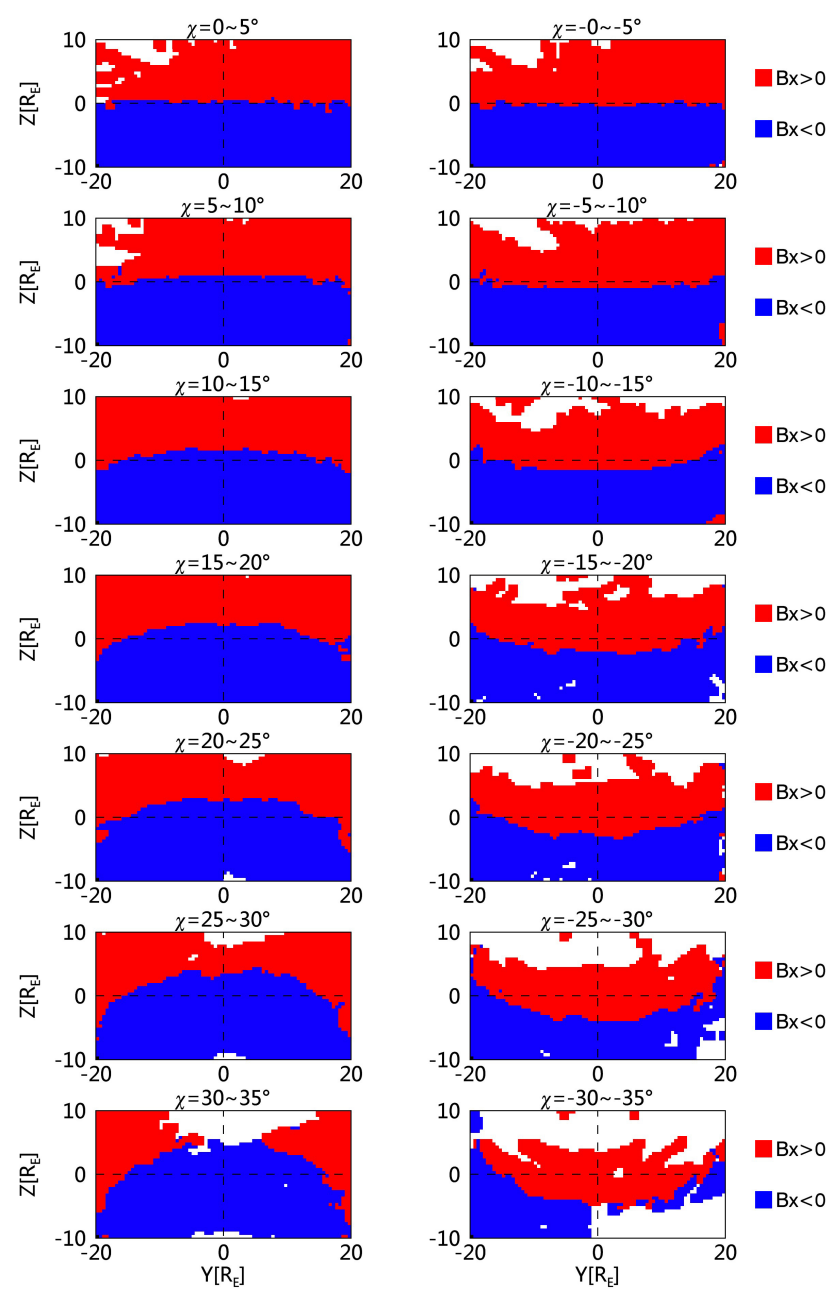

Figure 6. The sign of $B_{X}$ (in AGSM) in $0.5 R_{\mathrm{E}} \times 0.5 R_{\mathrm{E}}$ bins for a $40 R_{\mathrm{E}} \times 20 R_{\mathrm{E}} Y Z$ cross section in $5^{\circ}$ intervals of dipole tilt angle. All data are normalized to $X=-20 R_{\mathrm{E}}$ and a solar wind dynamic pressure of $2 \mathrm{nPa}$. Red (blue) indicates more positive (negative) values of $B_{X}$ in each bin. The neutral sheet is located between the red and blue areas.

Figure 8 shows the neutral sheet configuration in the $X Y$ cross section with the data of $-5 R_{\mathrm{E}}<Y<5 R_{\mathrm{E}}$. The fitting angle of slope and the uncertainty are labeled. While the neutral sheet has a displacement along $Z$, it tilts slightly along the tailward direction when the dipole angle increases. The larger the dipole tilt angle is, the more the neutral sheet tilts. Therefore, the dipole tilt angle affects not only the neutral sheet warping in the $Y Z$ cross section but also the tilting of the neutral sheet in the $X Y$ cross section.

For a small dipole tilt, the shape of the neutral sheet on the cross section is almost a straight line, and the fitting will make little sense. Therefore, the four sets of parameters, where $|\theta|$ is less than $10^{\circ}$, are ignored. The final parameters can be obtained by taking the average of the remaining 10 sets of parameters listed in Table 1, and the final parameters are listed in Table 2. 

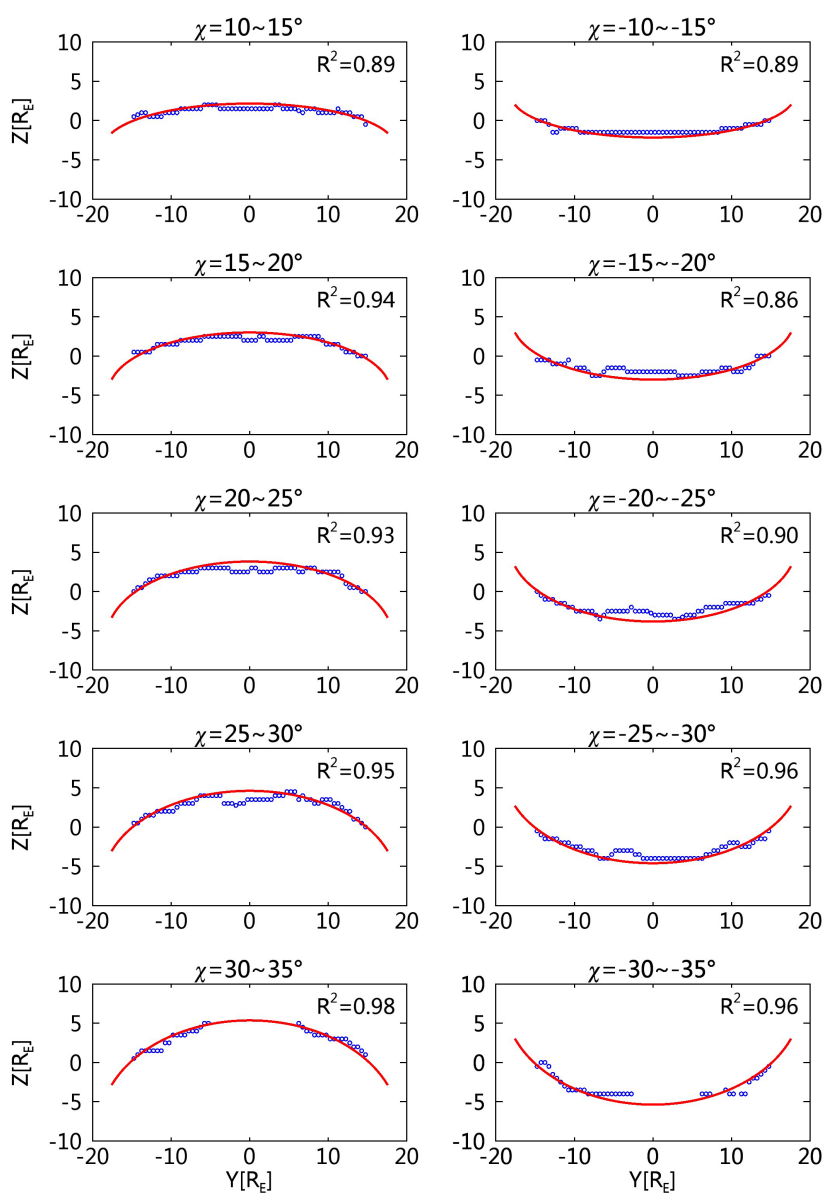

Figure 7. Fitting the neutral sheet locations in the $Y Z$ cross sections for different dipole tilt angles. The blue points give the positions $B_{X}=0$ (in AGSM), and the red lines are the best fits to these points.

The parameters listed in Table 2 have been normalized to eliminate the effect of solar wind dynamic pressure and magnetotail flaring. Thereby, the model function contains a scaling factor as follows:

$Z= \begin{cases}{\left[\left(H_{0}^{\prime}+D^{\prime}\right) \sqrt{1-\frac{Y^{2}}{Y_{0}^{\prime 2}}}-D^{\prime}\right] \sin \chi} & |Y|<Y_{0}^{\prime} \\ -D^{\prime} \sin \chi & |Y| \geq Y_{0}^{\prime},\end{cases}$

$$
H_{0}=9.98, \quad Y_{0}=18.44, \quad D=15.10,
$$

$H_{0}^{\prime}=H_{0} \cdot k_{\mathrm{sp}}, \quad Y_{0}^{\prime}=Y_{0} \cdot k_{\mathrm{sp}} \cdot k_{\mathrm{mf}}, \quad D^{\prime}=D \cdot k_{\mathrm{sp}}$

$$
k_{\mathrm{sp}}=\left(\frac{2}{P_{\mathrm{obs}}}\right)^{1 / 6}, \quad k_{\mathrm{mf}}=1.06 \arctan \left(\sqrt{\frac{10-X_{\mathrm{AGSM}}}{15.9}}\right),
$$

where $H_{0}^{\prime}, Y_{0}^{\prime}$, and $D^{\prime}$ are the three parameters of the displaced ellipse model. The parameters of $k_{\mathrm{sp}}$ and $k_{\mathrm{mf}}$ are the
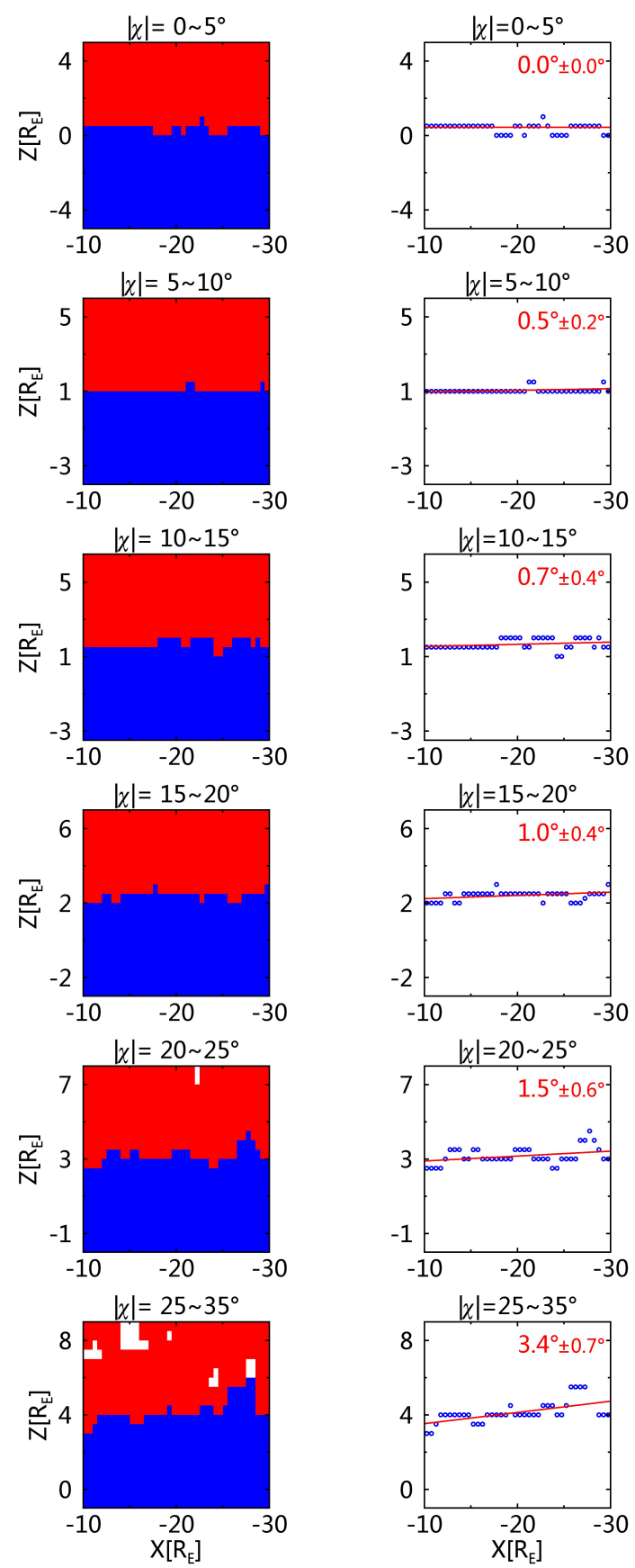

Figure 8. An illustration of the neutral sheet in the $X Y$ cross section for $5^{\circ}$ intervals of dipole tilt angle. Data of $-5 R_{\mathrm{E}}<Y<5 R_{\mathrm{E}}$ are chosen. To increase the data coverage, the negative dipole tilt angle data are combined with the positive angle data, and the data for a tilt greater than $25^{\circ}$ are combined also. The ratio of the $Z$ axis to the $X$ axis in data units is set as 2 .

correction factors of solar wind dynamic pressure and magnetotail flaring, respectively. $H_{0}, Y_{0}$, and $D$ are the normalized parameters under the solar wind dynamic pressure of $2 \mathrm{nPa}$ and the downtail distance of $20 R_{\mathrm{E}}$. 
Table 1. Parameters of the displaced ellipse model of the neutral sheet for $5^{\circ}$ intervals of dipole tilt, with a downtail distance of $20 R_{\mathrm{E}}$ and a solar wind dynamic pressure of $2 \mathrm{nPa}$.

\begin{tabular}{crrr}
\hline Dipole tilt & $H_{0}$ & $Y_{0}$ & $D$ \\
\hline$-35^{\circ}<\theta<-30^{\circ}$ & 9.981 & 18.969 & 15.100 \\
$-30^{\circ}<\theta<-25^{\circ}$ & 9.982 & 18.908 & 15.096 \\
$-25^{\circ}<\theta<-20^{\circ}$ & 9.978 & 18.226 & 15.102 \\
$-20^{\circ}<\theta<-15^{\circ}$ & 9.976 & 17.943 & 15.104 \\
$-15^{\circ}<\theta<-10^{\circ}$ & 9.977 & 18.066 & 15.106 \\
$10^{\circ}<\theta<15^{\circ}$ & 9.979 & 18.472 & 15.094 \\
$15^{\circ}<\theta<20^{\circ}$ & 9.977 & 17.942 & 15.121 \\
$20^{\circ}<\theta<25^{\circ}$ & 9.978 & 18.161 & 15.106 \\
$25^{\circ}<\theta<30^{\circ}$ & 9.980 & 18.656 & 15.101 \\
$30^{\circ}<\theta<35^{\circ}$ & 9.984 & 19.071 & 15.096 \\
\hline
\end{tabular}

Table 2. Final parameters of the displaced ellipse model of the neutral, with a downtail distance of $20 R_{\mathrm{E}}$ and a solar wind dynamic pressure of $2 \mathrm{nPa}$.

\begin{tabular}{lrr}
\hline Parameter & $\begin{array}{r}\text { Hammond } \\
\text { et al. (1994) }\end{array}$ & Present study \\
\hline$H_{0}$ & 9.57 & 9.98 \\
$Y_{0}$ & 21.48 & 18.44 \\
$D$ & 13.58 & 15.10 \\
\hline
\end{tabular}

A comparison with three prior studies is given in Fig. 9. All model curves are shown for a dipole tilt of $30^{\circ}$. The result of this study is shown by the blue line. The green, red and brown lines indicate the models of Fairfield (1980), Hammond et al. (1994), and Tsyganenko and Fairfield (2004), respectively. Except for the model of Fairfield (1980), which did not consider the effect of solar wind dynamic pressure and downtail distance, the other three models are shown for a downtail distance of $20 R_{\mathrm{E}}$ and a solar wind dynamic pressure of $2 \mathrm{nPa}$. As Fig. 9 shows, at the center, where the $Y$ axis value is zero, the height of the neutral sheet in this study is close to the prior models, a little lower than Fairfield (1980) and a little higher than Hammond et al. (1994) and Tsyganenko and Fairfield (2004). The difference becomes less when the dipole tilt decreases. Our hinging distance is a little greater than that of Hammond et al. (1994) and less than that given by Fairfield (1980). Our result is very similar to the result of Tsyganenko and Fairfield (2004), which indicates that the displaced ellipse model with suitable parameters are reliable to study the average of the neutral sheet shape.

\section{IMF dependence of the magnetotail neutral sheet}

The IMF has a bearing upon the shape of the neutral sheet (Russell, 1972, 1973). The IMF $B_{Y}$ can lead to a twisting of the magnetotail (Cowley, 1981), as well as the neutral sheet. The IMF $B_{Y}$-related twisting effect has been observed in prior studies (e.g., Slavin et al., 1983; Sibeck et al., 1985).

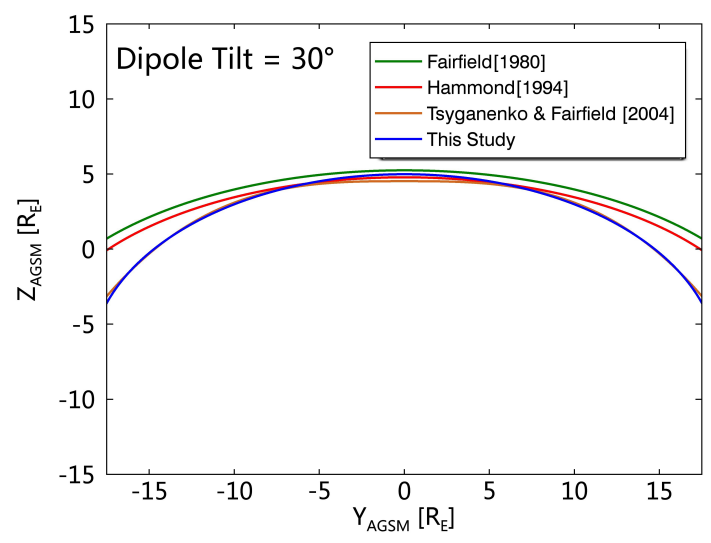

Figure 9. Comparison of the curves in the $Y Z$ cross section of the neutral sheet of three similar studies. Four neutral sheet models from three previous studies and this study are shown for a dipole tilt of $30^{\circ}$. The blue line shows the result of this study. The green line indicates the model of Fairfield (1980). The red line indicates the model of Hammond et al. (1994). The brown line, which is close to the blue line, represents the model of Tsyganenko and Fairfield (2004). Except for Fairfield (1980), the neutral sheet models are shown with a downtail distance of $20 R_{\mathrm{E}}$ and a solar wind dynamic pressure of $2 \mathrm{nPa}$.

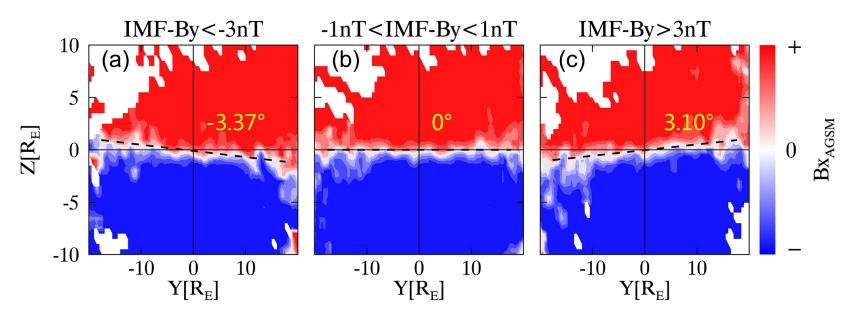

Figure 10. Illustration of the IMF- $B_{Y}$ related twisting effect on the neutral sheet for (a) IMF- $B_{Y}<-3 \mathrm{nT}$, (b) $-1 \mathrm{nT}<\mathrm{IMF}-B_{Y}<1 \mathrm{nT}$, and (c) IMF- $B_{Y}>3 \mathrm{nT}$. The white belt between red and blue indicates the shape of the neutral sheet. The black dotted lines are the fitting results of the neutral sheet, and the twisting angles are marked in the respective panel. In order to obtain a more pronounced visual effect, the ratio of the $Z$ axis to the $Y$ axis in data units is set as 2. It is shown that a counterclockwise (clockwise) twisting of the neutral sheet is observed, along the downtail direction, when the IMF- $B_{Y}$ is positive (negative).

Previous works (e.g., Owen et al., 1995; Maezawa and Hori, 1998) have also found that the IMF $B_{Y}$-related twisting becomes much larger during the periods of northward IMF.

In order to show a flat neutral sheet, only data with a low dipole tilt angle (absolute value less than $5^{\circ}$ ) are used. To statistically study the effect of large angle twisting on the neutral sheet under different levels of the IMF $B_{Y}$, the data with IMF $B_{Y}-8$ to $-3 \mathrm{nT}$ (negative), -1 to $1 \mathrm{nT}$ (zero), and 3 to $8 \mathrm{nT}$ (positive) are used in this section. Figure 10 shows the twisting effect of the IMF $B_{Y}$ on the neutral sheet (in order to obtain a more pronounced visual effect, the ratio of the $Z$ axis to the $Y$ axis in data units is set as 2). The white belt between 
Table 3. Summary of previous studies and comparison with the present study.

\begin{tabular}{|c|c|c|c|c|}
\hline & Fairfield (1980) & Hammond et al. (1994) & $\begin{array}{l}\text { Tsyganenko and } \\
\text { Fairfield (2004) }\end{array}$ & Present study \\
\hline Satellites & $\begin{array}{l}\text { IMP-6 } \\
\text { IMP-7 } \\
\text { IMP-8 }\end{array}$ & $\begin{array}{l}\text { IMP-7 } \\
\text { IMP-8 } \\
\text { ISEE-2 } \\
\text { OMNI }\end{array}$ & $\begin{array}{l}\text { Geotail } \\
\text { Polar } \\
\text { ACE } \\
\text { WIND } \\
\text { IMP-8 }\end{array}$ & $\begin{array}{l}\text { Cluster 1-4 } \\
\text { Geotail } \\
\text { TC-1 } \\
\text { THEMIS 1-5 } \\
\text { OMNI }\end{array}$ \\
\hline $\begin{array}{l}\text { Magnetic field } \\
\text { data }\end{array}$ & $\begin{array}{l}\text { IMP-6 } \\
1971-19739-12 \\
\text { IMP-7 } \\
1972.10-1973.3 \\
\text { IMP-8 } \\
1973.11-1974.11\end{array}$ & $\begin{array}{l}\text { IMP-7 } \\
1973 \\
\text { IMP-8 } \\
1978-1986 \\
\text { ISEE-2 } \\
1978 / 1979 / 1984 / 1986\end{array}$ & $\begin{array}{l}\text { Geotail } \\
\text { 1994-2002 } \\
\text { Polar } \\
\text { 1999-2001 }\end{array}$ & $\begin{array}{l}\text { Cluster 1-4 } \\
\text { 2001-2010 } \\
\text { Geotail } \\
\text { 1995-2013 } \\
\text { TC-1 } \\
\text { 2004-2007 } \\
\text { THEMIS } \\
\text { P1-P2 } \\
\text { 2007-2009 } \\
\text { P3-P5 } \\
\text { 2007-2013 }\end{array}$ \\
\hline Solar wind data & No & OMNI & $\begin{array}{l}\text { ACE } \\
\text { WIND } \\
\text { IMP-8 }\end{array}$ & OMNI \\
\hline Scaling & No & $\begin{array}{l}\text { Solar wind dynamic } \\
\text { pressure } \\
\text { Flaring of the magneto- } \\
\text { tail }\end{array}$ & No & $\begin{array}{l}\text { Solar wind dynamic } \\
\text { pressure } \\
\text { Flaring of the magneto- } \\
\text { tail }\end{array}$ \\
\hline Model & $\begin{array}{l}\text { The displaced ellipse } \\
\text { model }\end{array}$ & $\begin{array}{l}\text { The displaced ellipse } \\
\text { model }\end{array}$ & $\begin{array}{l}\text { A semi-empirical } \\
\text { model }\end{array}$ & $\begin{array}{l}\text { The displaced ellipse } \\
\text { model }\end{array}$ \\
\hline $\begin{array}{l}\text { Obtaining } \\
\text { parameters }\end{array}$ & $\begin{array}{l}\text { Minimizing the number } \\
\text { of mismatches between } \\
\text { the observed and pre- } \\
\text { dicted orientation of the } \\
\text { magnetic field on two } \\
\text { sides of the model }\end{array}$ & $\begin{array}{l}\text { Fitting the points } \\
\text { between bins with } \\
\text { different sign of } B_{X}\end{array}$ & $\begin{array}{l}\text { Minimizing the number } \\
\text { of mismatches between } \\
\text { the observed and pre- } \\
\text { dicted orientation of the } \\
\text { magnetic field on two } \\
\text { sides of the model }\end{array}$ & $\begin{array}{l}\text { Fitting the points } \\
\text { between bins with } \\
\text { different sign of } B_{X}\end{array}$ \\
\hline Coordinate system & AGSM & AGSM & GSW & AGSM \\
\hline
\end{tabular}

red and blue area indicates the shape of the neutral sheet. As expected, looking along the downtail direction, the neutral sheet is twisting clockwise for a negative IMF $B_{Y}$ and counterclockwise for positive IMF $B_{Y}$. Here we find a clockwise twist of $3.37^{\circ}$ for the IMF $B_{Y}$ between -8 and $-3 \mathrm{nT}$ and a counterclockwise twist of $3.10^{\circ}$ for the IMF $B_{Y}$ between 3 and $8 \mathrm{nT}$. Furthermore, the IMF $B_{Z}$ also plays a significant role in the neutral sheet twisting. As shown in Fig. 11, we separate Fig. 10a and c into positive and negative IMF $B_{Z}$ periods. For the IMF $B_{Y}$ between -8 and $-3 \mathrm{nT}$, one can find a clockwise twist $\sim 4.40^{\circ}$ for positive IMF $B_{Z}$, and $\sim 2.73^{\circ}$ for negative IMF $B_{Z}$; for the IMF $B_{Y}$ between 3 and $8 \mathrm{nT}$, a counterclockwise twist $\sim 3.37^{\circ}$ is found for positive IMF $B_{Z}$, and $\sim 2.20^{\circ}$ for negative IMF $B_{Z}$. This indicates that a northward IMF can result in a greater twisting of the neutral sheet in the near tail.

\section{Discussion and conclusions}

Our results show that the displaced ellipse model of the neutral sheet has a greater degree of curve than that of Hammond et al. (1994) and is close to the model of Tsyganenko and Fairfield (2004). The position of the hinging point in this study is close to the findings in the prior studies (Hammond et al., 1994; Tsyganenko and Fairfield, 2004). As shown in Fig. 9, the curves in the cross section of $Y Z$ are plotted with a very large dipole tilt of $30^{\circ}$ to make the difference clearer. The curves will be closer to each other for a smaller dipole tilt. Fairfield (1980) did not normalize the data, which have a significant effect on the result, so the curve of Fairfield (1980) is just for reference.

As listed in Table 3, there are some comparisons of this work with prior works. In the past few decades, more satel- 

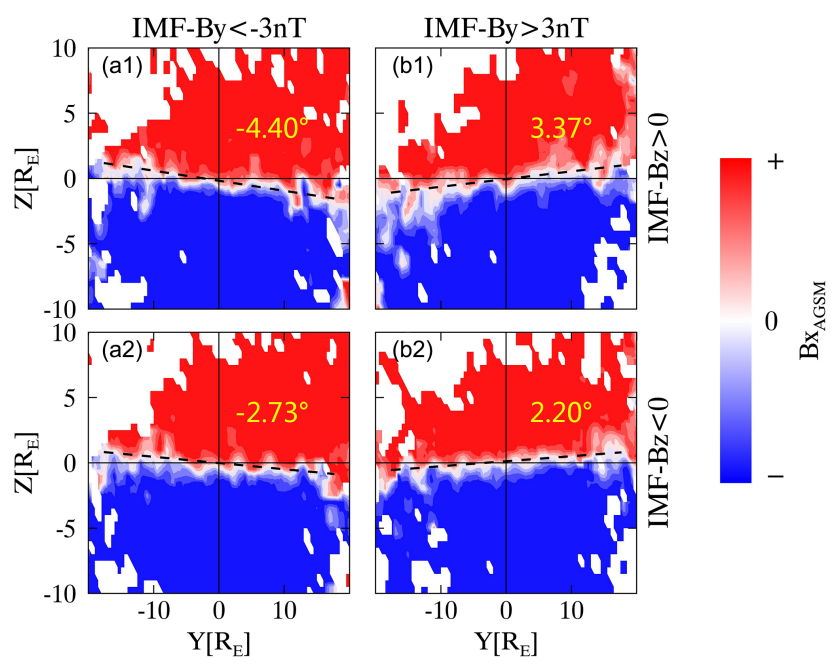

Figure 11. Illustration of the IMF- $B_{Z}$ effect on the neutral sheet twisting for (a1) IMF- $B_{Y}<-3 \mathrm{nT}$ and IMF- $B_{Z}>0 \mathrm{nT}$, (b1) IMF$B_{Y}>3 \mathrm{nT}$ and IMF- $B_{Z}>0 \mathrm{nT}$, (a2) IMF- $B_{Y}<-3 \mathrm{nT}$ and IMF$B_{\mathrm{Z}}<0 \mathrm{nT}$, and (b2) IMF- $B_{Y}>3 \mathrm{nT}$ and IMF- $B_{\mathrm{Z}}<0 \mathrm{nT}$. It is shown that a northward IMF can result in a larger twisting angle of the near-tail neutral sheet.

lites have become available and more data can be obtained. We define a satellite year as the number of years of data. However, satellites with similar orbits are counted only once. For this study, the four Cluster satellites count as one, and THEMIS P3-P5 should be counted as one, too. Thereby, our study spans 46 satellite years, containing 10 years of Cluster, 19 years of Geotail, 4 years of TC- 1 , and $3+3+7$ years of THEMIS (compared with 9 satellite years in the study of Fairfield, 1980, 14 satellite years in the study of Hammond et al., 1994, and 12 satellite years of Tsyganenko and Fairfield, 2004). As in the investigations of Fairfield (1980) and Hammond et al. (1994), the data are converted to the AGSM coordinate system in this work. Tsyganenko and Fairfield (2004) converted the data to the GSW (geocentric solar wind) coordinate system. The difference between the AGSM coordinate system and the GSW coordinate system is the direction of the $X$ axis. The $X$ axis in the AGSM coordinate system is antiparallel to the average solar wind flow, while the $X$ axis in the GSW coordinate system is antiparallel to the observed solar wind flow.

The effect of solar wind dynamic pressure and magnetotail flaring on the shape of the neutral sheet is substantial. Thus all data are scaled to the same solar wind dynamic pressure and downtail distance to eliminate their effects. We use a statistical method to investigate the average position of the neutral sheet with grids (bins) in the $Y Z$ cross section, instead of simply recording where the $X$ component of the magnetic field changes sign. The bin method offers a superb solution to solve the problem of undersampling and increases the accuracy of the result. Fairfield (1980) parameterized the model by minimizing the number of mismatches between the observed and predicted orientation of the magnetic field on two sides of the neutral sheet, same as Tsyganenko and Fairfield (2004). The method of Fairfield (1980) can also solve the problem of undersampling effectively, but it is hard to produce visualized images like Figs. 6, 7, and 8. Therefore, the bin method is a good choice.

Figure 6 illustrates the change in the average shape of the neutral sheet with different dipole tilt angle. Obviously, a positive dipole tilt leads to a northern curve and a negative dipole tilt leads to a southern curve. In addition, the larger the dipole tilt is, the greater the curvature is. As Fig. 6 shows, the shape of the curve of the neutral sheet in the $Y Z$ cross section is almost a semi-ellipse, and the flanks of the neutral sheet extend under the $X Y$ plane. The results are consistent with expectations. To balance the magnetic pressure in two lobes, the neutral sheet has a displacement along $Z$ with curving.

In this investigation, we find that the dipole tilt angle has a global influence on the neutral configuration. It affects not only the warping of the neutral sheet in the $Y Z$ cross section but also the tilting in the $X Y$ cross section. Furthermore, we observe the IMF $B_{Y}$ twisting effect on the neutral sheet. As shown in Fig. 10, the neutral sheet twists clockwise for a negative IMF $B_{Y}$ and counterclockwise for a positive IMF $B_{Y}$. It is considered that the farther the distance from Earth, the larger the degree of the IMF $B_{Y}$ twisting (Cowley, 1980; Tsyganenko and Fairfield, 2004). The IMF $B_{Y}$ twisting effect in the distant magnetotail has been observed in some prior studies (Slavin et al., 1983; Sibeck et al., 1985; Owen et al., 1995; Maezawa and Hori, 1998). In this study, the observation of the IMF $B_{Y}$ twisting effect focused on the near tail, between $X=-10 R_{\mathrm{E}}$ and $-35 R_{\mathrm{E}}$. It is observed that the larger the IMF $B_{Y}$, the larger the twisting angle. However, because of the lack of data for large IMF $B_{Y}$, it could not be accurate enough to investigate the quantitative relation between the twisting angle and the IMF $B_{Y}$ in this observation. In the near tail, we can also observe a larger twisting angle for northward IMF $B_{Z}$ than for southward, as which has been observed in the distant tail (e.g., Owen et al., 1995; Maezawa and Hori, 1998).

There have been a series of works reporting the model of the magnetotail neutral sheet (e.g., Fairfield, 1980; Hammond et al., 1994; Tsyganenko and Fairfield, 2004). Recently, a new quantitative model of the shape of the magnetospheric equatorial current sheet has been developed, taking the dipole tilt angle, the solar wind ram pressure, and transverse components of the IMF into consideration (Tsyganenko et al., 2015). Tsyganenko et al. (2015) defined the current sheet as the location where the observed magnetic field reverses its radial component. This model provides a global view of the current sheet. Compared to the model of Tsyganenko et al. (2015), we provide a reliable average neutral sheet shape and position with a large database. Our results can be a significant reference to the model of the neutral sheet. 
Acknowledgements. This work was supported by NSFC grants 41574173, 41421063, and 41474144 in China and by EU FP7 grant 263325 - ECLAT and the Austrian Science Fund FWF P23862-N16 and Fund P24740-N27 in Austria. We are grateful to the teams and PIs of all experiments whose data were used in this study. We appreciate Cluster Active Archive at http://caa.estec.esa.int/ and the THEMIS data center of Berkeley at http://themis.ssl.berkeley.edu/ for the data used in this study. The TC-1 FGM and PGP data were provided by the Austrian DSP Data Center at http://dione. iwf.oeaw.ac.at/ddms/, and the Geotail MGF data and the interplanetary data of OMNI were obtained from CDAWeb online facility at http://cdaweb.gsfc.nasa.gov/.

The topical editor, C. Owen, thanks H. Lühr and one anonymous referee for help in evaluating this paper.

\section{References}

Auster, H. U., Glassmeier, K., Magnes, W., Aydogar, O., Baumjohann, W., Constantinescu, D., Fischer, D., Fornacon, K., Georgescu, E., Harvey, P., Hillenmaier, O., Kroth, R., Ludlam, M., Narita, Y., Nakamura, R., Okrafka, K., Plaschke, F., Richter, I., Schwarzl, H., Stoll, B., Valavanoglou, A., and Wiedemann, M.: The THEMIS fluxgate magnetometer, Space Sci. Rev., 141, 235-264, doi:10.1007/s11214-008-9365-9, 2008.

Balogh, A., Carr, C. M., Acuña, M. H., Dunlop, M. W., Beek, T. J., Brown, P., Fornacon, K.-H., Georgescu, E., Glassmeier, K.H., Harris, J., Musmann, G., Oddy, T., and Schwingenschuh, K.: The Cluster Magnetic Field Investigation: overview of in-flight performance and initial results, Ann. Geophys., 19, 1207-1217, doi:10.5194/angeo-19-1207-2001, 2001.

Baumjohann, W. and Treumann, R. A.: Basic Space Plasma Physics (Revised Edition), Imperial College Press, London, UK, 2012.

Carr, C., Brown, P., Zhang, T. L., Gloag, J., Horbury, T., Lucek, E., Magnes, W., O’Brien, H., Oddy, T., Auster, U., Austin, P., Aydogar, O., Balogh, A., Baumjohann, W., Beek, T., Eichelberger, H., Fornacon, K.-H., Georgescu, E., Glassmeier, K.-H., Ludlam, M., Nakamura, R., and Richter, I.: The Double Star magnetic field investigation: instrument design, performance and highlights of the first year's observations, Ann. Geophys., 23, 2713-2732, doi:10.5194/angeo-23-2713-2005, 2005.

Cowley, S. W. H.: Magnetospheric asymmetries associated with the y-component of the IMF, Planet. Space Sci., 29, 79-96, 1981.

Fairfield, D.: A statistical determination of the shape and position of the geomagnetic neutral sheet, J. Geophys. Res., 85, 775-780, doi:10.1029/JA085iA02p00775, 1980.

Hammond, C. M., Kivelson, M. G. and Walker, R. J.: Imaging the effect of dipole tilt on magnetotail boundaries, J. Geophys. Res., 99, 6079-6092, doi:10.1029/93JA01924, 1994.
Howe Jr., H. C. and Binsack, J. H.: Explorer 33 and 35 plasma observations of magnetosheath flow, J. Geophys. Res., 77, 3334 3344, doi:10.1029/JA077i019p03334, 1972.

Kokubun, S., Yamamoto, T., Acuña, M. H., Hayashi, K., Shiokawa, K., and Kawano, H.: The GEOTAIL magnetic field experiment, J. Geomagn. Geoelectr., 46, 7-21, 1994.

Maezawa, K. and Hori, T.: The Distant Magnetotail: Its Structure, IMF Dependence, and Thermal Properties, in New Perspectives on the Earth's Magnetotail, American Geophysical Union, Washington, D.C., USA, 1-19, doi:10.1029/GM105p0001, 1998.

Murayama, T.: Spatial distribution of energetic electrons in the geomagnetic tail, J. Geophys. Res., 71, 5547-5557, doi:10.1029/JZ071i023p05547, 1966.

Ness, N. F.: The Earth's magnetic tail, J. Geophys. Res., 70, 2989 3005, doi:10.1029/JZ070i013p02989, 1965.

Owen, C. J., Slavin, J. A., Richardson, I. G., Murphy, N., and Hynds, R. J.: Average motion, structure and orientation of the distant magnetotail determined from remote sensing of the edge of the plasma sheet boundary layer with $\mathrm{E}>35 \mathrm{keV}$ ions, J. Geophys. Res.-Atmos., 100, 185-204, doi:10.1029/94JA02417, 1995.

Russell, C. T.: The configuration of the magnetosphere, in: Critical Problems of Magnetospheric Physics, edited by: Dyer, E. R., p. 1, IUCSTP Secretariat, National Academy of Sciences, Washington DC, USA, 1972.

Russell, C. T.: Comments on the paper "The internal structure of the geomagnetic neutral sheet”, by K. Schindler and N. F. Ness, J. Geophys. Res., 78, 7576-7579, doi:10.1029/JA078i031p07576, 1973.

Russell, C. T. and Brody, K. I.: Some remarks on the position and shape of the neutral sheet, J. Geophys. Res., 72, 6104-6106, doi:10.1029/JZ072i023p06104, 1967.

Sibeck, D. G., Siscoe, G. L., Slavin, J. A., Smith, E. J., Tsurutani, B. T., and Lepping, R. P.: The distant magnetotail's response to a strong interplanetary magnetic field By: Twisting, flattening, and field line bending, J. Geophys. Res., 90, 4011-4019, doi:10.1029/JA090iA05p04011, 1985.

Slavin, J. A., Tsurutani, B. T., Smith, E. J., Jones, D. E., and Sibeck, D. G.: Average configuration of the distant $(<220 \mathrm{Re})$ magnetotail: Initial ISEE-3 magnetic field results, Geophys. Res. Lett., 10, 973-976, doi:10.1029/GL010i010p00973, 1983.

Tsyganenko, N. A. and Fairfield, D. H.: Global shape of the magnetotail current sheet as derived from Geotail and Polar data, J. Geophys. Res., 109, A03218, doi:10.1029/2003JA010062, 2004.

Tsyganenko, N. A., Andreeva, V. A., and Gordeev, E. I.: Internally and externally induced deformations of the magnetospheric equatorial current as inferred from spacecraft data, Ann. Geophys., 33, 1-11, doi:10.5194/angeo-33-1-2015, 2015. 